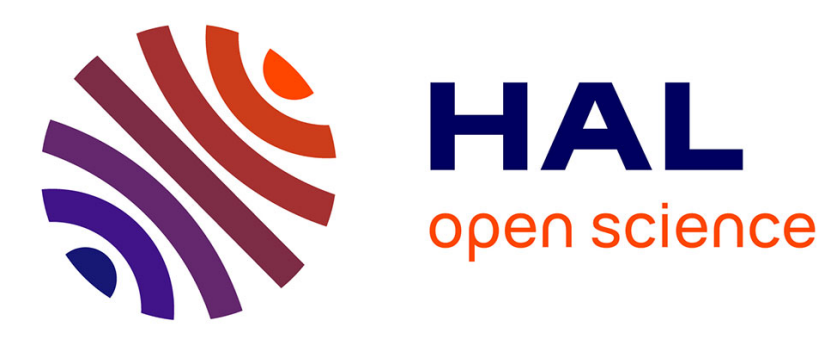

\title{
Cooperative differentiation through clustering in multicellular populations
}

\author{
A. Koseska, E. Ullner, E. Volkov, J. Kurths, J. García-Ojalvo
}

\section{To cite this version:}

A. Koseska, E. Ullner, E. Volkov, J. Kurths, J. García-Ojalvo. Cooperative differentiation through clustering in multicellular populations. Journal of Theoretical Biology, 2010, 263 (2), pp.189. 10.1016/j.jtbi.2009.11.007 . hal-00566753

\section{HAL Id: hal-00566753 https://hal.science/hal-00566753}

Submitted on 17 Feb 2011

HAL is a multi-disciplinary open access archive for the deposit and dissemination of scientific research documents, whether they are published or not. The documents may come from teaching and research institutions in France or abroad, or from public or private research centers.
L'archive ouverte pluridisciplinaire HAL, est destinée au dépôt et à la diffusion de documents scientifiques de niveau recherche, publiés ou non, émanant des établissements d'enseignement et de recherche français ou étrangers, des laboratoires publics ou privés. 


\section{Author's Accepted Manuscript}

Cooperative differentiation through clustering in multicellular populations

A. Koseska, E. Ullner, E. Volkov, J. Kurths, J. García-Ojalvo

PII:

S0022-5193(09)00533-5

DOI: doi:10.1016/j.jtbi.2009.11.007

Reference: YJTBI 5772

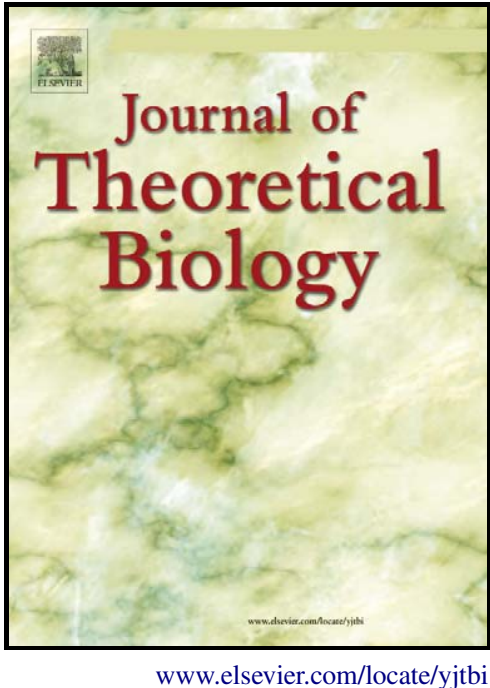

To appear in: Journal of Theoretical Biology

Received date: $\quad 10$ July 2009

Revised date: $\quad 23$ October 2009

Accepted date: $\quad 10$ November 2009

Cite this article as: A. Koseska, E. Ullner, E. Volkov, J. Kurths and J. García-Ojalvo, Cooperative differentiation through clustering in multicellular populations, Journal of Theoretical Biology, doi:10.1016/j.jtbi.2009.11.007

This is a PDF file of an unedited manuscript that has been accepted for publication. As a service to our customers we are providing this early version of the manuscript. The manuscript will undergo copyediting, typesetting, and review of the resulting galley proof before it is published in its final citable form. Please note that during the production process errors may be discovered which could affect the content, and all legal disclaimers that apply to the journal pertain. 


\title{
Cooperative differentiation through clustering in multicellular populations
}

\author{
A. Koseska*,a , E. Ullner ${ }^{\mathrm{b}, \mathrm{c}, \mathrm{d}, \mathrm{e}}$, E. Volkov ${ }^{\mathrm{f}}$, J. Kurths $^{\mathrm{g}, \mathrm{h}}$, J. García-Ojalvo $^{\mathrm{d}}$ \\ ${ }^{a}$ Center for Dynamics of Complex Systems, University of Potsdam, D-14469 Germany \\ ${ }^{b}$ Institute for Complex Systems and Mathematical Biology, King College, \\ University of Aberdeen, Aberdeen AB24 3UE, United Kingdom \\ ${ }^{c}$ Institute of Medical Sciences, Forresterhill, \\ University of Aberdeen, Aberdeen AB25 2DZ, United Kingdom \\ ${ }^{d}$ Departament de Física i Enginyeria Nuclear, Universitat Politècnica de Catalunya, \\ Colom 11, E-08222 Terrassa, Spain \\ e Institute for Theoretical Biology (ITB), Humboldt University, Invalidenstr. 43, \\ D-10115 Berlin, Germany \\ ${ }^{f}$ Department of Theoretical Physics, Lebedev Physical Inst., Leninskii 53, Moscow, Russia \\ ${ }^{g}$ Institute of Physics, Humboldt University Berlin, D-10099 Germany \\ ${ }^{h}$ Potsdam Institute for Climate Impact Research, D-14412 Germany
}

\section{Abstract}

The coordinated development of multicellular organisms is driven by intercellular communication. Differentiation into diverse cell types is usually associated with the existence of distinct attractors of gene regulatory networks, but how these attractors emerge from cell-cell coupling is still an open question. In order to understand and characterize the mechanisms through which coexisting attractors arise in multicellular systems, here we systematically investigate the dynamical behavior of a population of synthetic genetic oscillators coupled by chemical means. Using bifurcation analysis and numer-

\footnotetext{
*Corresponding author

Email addresses: koseska@yahoo.com (A. Koseska), e.ullner@abdn.ac.uk (E. Ullner), volkov@td.lpi.ru (E. Volkov), kurths@pik-potsdam.de (J. Kurths), jordi.g.ojalvo@upc.edu (J. García-Ojalvo)
} 
ical simulations, we identify various attractors and attempt to deduce from these findings a way to predict the organized collective behavior of growing populations. Our results show that dynamical clustering is a generic property of multicellular systems. We argue that such clustering might provide a basis for functional differentiation and variability in biological systems.

Key words:

Multicellular systems, clustering, collective behavior, inhibitory cell-to-cell communication, cellular differentiation 


\section{1. Introduction}

2 The coordinated behavior in multicellular systems results from a coop-

3 erative response arising from an integrated exchange of information through

4 cell-cell communication. Various mechanisms for intercellular coupling have

5 been identified in nature, basically relying on the broadcasting of individ-

6 ual cellular states to neighboring cells via intercellular signals, which are

7 further integrated to generate a global system's response (Heinlein (2002);

8 Perbal (2003)). It is known, for instance, that bacteria display various types

9 of collective behavior driven by a type of chemical cell-cell communication

10 mechanism known as quorum sensing (Taga and Bassler (2003)). This ability

11 of living systems is an absolute requisite to ensure an appropriate and ro-

12 bust global cellular response of an organism in a noisy environment. Hence,

13 characterizing the dynamics of multicellular systems should lead to an im-

14 provement of our knowledge about cellular behavior and biological mecha-

15 nisms that occur on a population-wide scale, such as cellular differentiation,

16 adaptability of the system to different environment conditions, etc.

17 In order to understand the basic mechanisms of cell-to-cell cooperative be-

18 havior, several theoretical models have been successfully developed and inves-

19 tigated by studying both natural and synthetic genetic networks (McMillen

20 et al. (2002); Taga and Bassler (2003); Kuznetsov et al. (2004); García-Ojalvo

21 et al. (2004); Ullner et al. (2007); Balagadde et al. (2008); Tanouchi et al.

22 (2008)). A synchronization scheme has been proposed, for instance, in an

23 artificial network of synthetic genetic oscillators that produces and responds

24 to a specific, small signaling molecule (acylated homoserine lactone), known

25 as an autoinducer $(A I)$ (García-Ojalvo et al. (2004)). This small molecule is 
26 free to diffuse through the cell membrane, which provides a means for chem-

27 ical communication between neighboring cells. The resulting synchronized

28 behavior leads to a macroscopic genetic clock. By further manipulations of

29 this synthetic network, we were able to show (Ullner et al. (2007, 2008))

30 that this communication scheme can be re-engineered to produce a very di-

31 verse dynamics and exhibit a high adaptability typical to natural systems.

32 Other modification of the same network (García-Ojalvo et al. (2004)) was also

33 published recently (Zhou et al. (2008)), but it still waits futher dynamical

34 investigations.

35 In this paper, we investigate systematically the global cooperative behav-

36 ior of a population of synthetic genetic oscillators called repressilators, cou-

37 pled via quorum sensing mechanism (Ullner et al. (2007)), and thus showing

38 the emergence of a rich variety of clustering behavior that might be inter-

39 preted as a mechanism of dynamical differentiation. Such an interpretation

40 was pioneered by Turing (Turing (1952)) in his investigations of inhomo-

41 geneous steady states in reaction-diffusion systems, and has been further

42 extended by Kaneko (Kaneko and Yomo (1994)), who proposed clustering in

43 coupled map dynamics as a physical background of biological differentiation.

44 Moreover, it was recently shown (Nakajima and Kaneko (2008)) that bifur-

45 cations driven by cell-cell interaction may mediate differentiation processes.

46 Here we use numerical simulations and bifurcation analysis to study the

47 dynamics of a population of coupled genetic oscillators for increasing sizes

48 of the population, ranging from a two-cell to a multicellular system, thus

49 proposing a general explanation for the emergence of cooperative behavior

50 in large cellular systems. In our previous work (Ullner et al. (2007, 2008)) 
51 we investigated and defined the necessary coupling conditions leading to a

52 complex dynamical behavior in the system, and furthermore clasified its dy-

53 namical structure using a minimal system of $N=2$ oscillators. In addition to

54 those earlier results, here we show that the extended system exhibits various

55 attractors with complex phase relations, and through their characterization

56 we attempt to (i) deduce the underlying mechanism that determines the most

57 likely visited dynamical regimes, and (ii) identify stable cluster distributions,

58 in order to predict the behavior of the system on a global scale. The bifurca-

59 tion analysis and numerical investigations presented here also aim to charac-

60 terize the robustness of the dynamical structure of the system with respect

61 to parameter variations, and relate these findings to biological processes. We

62 stress here the importance of investigating dynamical clustering in multicel-

63 lular populations with cell-cell communication, since such coupling generates

64 qualitatively new cellular states different from the single-cell dynamics, thus

65 providing the basis for functional differentiation and variability.

66 Moreover, in (Ullner et al. (2008)) we identified a biologically relevant

67 parameter interval where chaotic behavior of the coupled genetic units was

68 observed. As previously suggested, this could implicate chaos as an addi-

69 tional source of uncertainty in gene expression, drawing attention on possi-

70 ble alternative sources of uncertainty in genetic networks, besides the already

71 well-established ones. It is therefore important to investigate the dynami-

72 cal behavior of cell populations in the chaotic regime, and identify possible

73 groupings of genetic oscillators and their relations within, as a base for en-

74 visioning an experimental protocol to detect chaotic behavior in synthetic

75 genetic networks. 


\section{2. Model of a synthetic multicellular system}

77 The model considered here consists of a population of repressilators cou78 pled via quorum-sensing mechanism as proposed in (Ullner et al. (2007))

79 The repressilator consists of three genes whose protein products repress the

80 transcription of each other in a cyclic way (Elowitz and Leibler (2000)). In its

81 original experimental implementation, the gene lacI expresses protein LacI,

82 which inhibits transcription of the gene tetR. The product of the latter, Tet $R$,

83 inhibits transcription of the gene $c I$. Finally, the protein product $C I$ of the

84 gene $c I$ inhibits expression of lacI and completes the cycle (see Fig. 1). An

85 additional feedback loop involving the two proteins $L u x I$ and $L u x R$, which

86 might be placed on a separate plasmid, realizes the cell-to-cell communication

87 (McMillen et al. (2002); You et al. (2004); García-Ojalvo et al. (2004)). LuxI

88 is responsible for the biosynthesis of a small signaling molecule, known as au-

89 toinducer $(A I)$, which diffuses through the cell membrane and thus provides

90 a means of intercellular communication. By forming a stable $A I-L u x R$

91 complex, the transcription of a second copy of the repressilator gene lacI is

92 activated. Placing the gene luxI under inhibitory control of the repressilator

93 protein TetR (Fig. 1) introduces a rewiring between the repressilator and

94 the quorum sensing through an additional loop, which competes with the

95 overall negative feedback loop along the repressilator ring and results in an

96 inhibitory, phase-repulsive intercellular coupling.

The $m R N A$ dynamics is described by the following Hill-type kinetics with Hill coefficient $n$ :

$$
\dot{a}_{i}=-a_{i}+\frac{\alpha}{1+C_{i}^{n}}
$$




$$
\begin{aligned}
\dot{b}_{i} & =-b_{i}+\frac{\alpha}{1+A_{i}^{n}} \\
\dot{c}_{i} & =-c_{i}+\frac{\alpha}{1+B_{i}^{n}}+\kappa \frac{S_{i}}{1+S_{i}}
\end{aligned}
$$

where the subindex $i$ denotes the cell $(i=1, . ., N, N$ being the total number of cells in the ensemble), and $a_{i}, b_{i}$ and $c_{i}$ represent the concentrations of $m R N A$ molecules transcribed from tetR, $c I$ and lacI, respectively. The model is made dimensionless by measuring time in units of the $m R N A$ lifetime (assumed equal for all genes) and the $m R N A$ and protein levels in units of their Michaelis constants (assumed equal for all genes). The $m R N A$ concentrations are additionally rescaled by the ratio of their protein degradation (different among the genes) and translation rates (assumed equal for all genes). After rescaling, $\alpha$ is the dimensionless transcription rate in the absence of a repressor, $\kappa$ is the maximum transcription rate of the Lux $R$ promoter, and the parameters $\beta_{a, b, c}$ describe the ratios between the $m R N A$ and protein lifetimes (inverse degradation rates). We assume different lifetime ratios for the protein $/ m R N A$ pairs, which results in a weak relaxator-like dynamics of the repressilator (Ullner et al. (2007)). $A_{i}, B_{i}$, and $C_{i}$ denote the concentration of the proteins TetR, CI, and LacI, whose dynamical behavior is given by:

$$
\begin{aligned}
\dot{A}_{i} & =\beta_{a}\left(a_{i}-A_{i}\right) \\
\dot{B}_{i} & =\beta_{b}\left(b_{i}-B_{i}\right) \\
\dot{C}_{i} & =\beta_{c}\left(c_{i}-C_{i}\right)
\end{aligned}
$$

Assuming equal lifetimes and dynamics for both the CI and LuxI proteins (since they are both repressed by TetR), we use the same variable to 
describe the dynamics of both proteins. The $A I$ concentration $S_{i}$ in the $i-t h$ cell (rescaled additionally by its Michaelis constant) is proportional to $B_{i}$, i.e. the concentration of LuxI in it, and is further affected by an intracellular degradation and diffusion toward or from the intercellular space:

$$
\begin{aligned}
\dot{S}_{i} & =-k_{s 0} S_{i}+k_{s 1} B_{i}-\eta\left(S_{i}-S_{e}\right) \\
S_{e} & =Q \bar{S} \\
\bar{S} & =\frac{1}{N} \sum_{i=1}^{N} S_{i}
\end{aligned}
$$

97 The diffusion coefficient $\eta$ depends on the permeability of the membrane 98 to the autoinducer. Due to the fast diffusion of the extracellular $A I\left(S_{e}\right)$ 99 compared to the repressilator period, we can apply the quasi-steady-state 100 approximation to the dynamics of the external $A I$ and replace it by the mean 101 field of the internal $A I, \bar{S}$. The parameter $Q$ is defined as $Q=\frac{\delta N / V_{e x t}}{k_{s e}+\delta N / V_{e x t}}$ 102 (García-Ojalvo et al. (2004)), with $N$ being the total number of cells in 103 the ensemble, $V_{\text {ext }}$ the total extracellular volume, $k_{s e}$ the extracellular $A I$ 104 degradation rate, and $\delta$ the product of the membrane permeability and the 105 surface area. In more general terms, $Q$ is proportional to the cell density, 106 and can be varied in a controlled way between 0 and 1 in experiment, thus 107 making it a reasonable choice to follow the dynamical changes of the system 108 with respect to $Q$.

109 Previous investigations carried on a minimal system of two repressilators 110 coupled via repulsive cell-to-cell communication (Ullner et al. (2008)) have 111 identified a variety of collective regimes, including constant level protein pro112 duction (homogeneous steady state solution, HSS, Fig. 2a), an inhomoge113 neous steady state characterized by different stationary protein levels (IHSS, 
114 Fig. 2b), and self-sustained oscillations. Within the latter case, we have iden115 tified out-of-phase oscillations with different phase shifts (see e.g. Fig. 2c, for

116 which the phase shift is $\frac{\pi}{2}$ ), as well as a complex inhomogeneous limit cycle 117 (IHLC) characterized by one cell exhibiting very small oscillations around 118 a high mean protein level, whereas the second cell oscillates in the vicinity 119 of the steady state with an amplitude just slightly smaller than that of an 120 isolated oscillator (Fig. 2d).

121 The previous results correspond to a fixed value $N=2$. However, in 122 vivo bacterial colonies proliferate and expand. In order to understand and 123 characterize the cooperative behavior in growing populations, it is certainly of 124 outmost significance to investigate the influence of the size of the population 125 on its dynamics. To that end, we performed a simple numerical experiment: 126 we computed 1000 time series with different random initial conditions for 127 a minimal $(N=2$ cells), and a system with intermediate size $(N=18)$, 128 using a uniform distribution in the range [0,220] for the $m R N A$ and protein 129 initial conditions and $[0,1.2]$ for the $A I$ initial conditions. Figure 3 shows 130 the histograms of detectable stable regimes in both systems for increasing 131 coupling coefficient $Q$.

132 As shown in Fig. 3, already a small increase in the system size (from $133 N=2$ to $N=18$ cells) alters the balance between the coexisting regimes: 134 the stability regions of IHLC and IHSS are significantly increased at the 135 expense of the HSS. This fact underlines the connection between the size of 136 the population and its dynamical behavior, implying that a detailed analysis 137 of these correlations is necessary in order to reveal and formulate (predict) a 138 general statement about the cooperative behavior of growing populations. 


\section{3. Clustering in the inhomogeneous regimes}

140 It is well known that genetically identical cells may exhibit diverse phe141 notypic states even under almost identical environmental conditions. Thus, 142 populations comprised of identical cellular units can display heterogeneity, 143 manifested by the existence of several subgroups or clusters where cells ex-

144 hibit organized collective behavior, with or without complex relations among

145 them. The size of the population plays a crucial role in determining which

146 dynamical behavior is most likely to be dominant, depending of course on

147 the environmental conditions as well as on the coupling strengths.

148 We now show that the parameter stability intervals for given solutions 149 increase significantly as a function of $N$, with respect to the equivalent stabil-

150 ity intervals in the minimal model of two coupled cells (Ullner et al. (2008)).

151 These changes in the dynamical structure of the model occur in general

152 due to the increased possibility for cluster formation in growing populations.

153 Clustering can be defined as a stable dynamical state characterized by the

154 coexistence of several subgroups where the oscillators exhibit identical (or 155 nearly identical) behavior. Clustering is a well known property, especially

156 for globally coupled systems, and has been investigated for identical phase

157 (Golomb et al. (1992); Okuda (1993)), salt-water (Miyakawa and Yamada

158 (2001)) or electrochemical oscillators (Wang et al. (2001); Kiss and Hudson 159 (2003)), in synthetic genetic networks (Koseska et al. (2007)), and in popu-

160 lations of chaotic oscillators (Kuznetsov and Kurths (2002); Manruiba and

161 Mikhailov (1999); Osipov et al. (2007)), among other cases. The presence of

162 clustering and the complex phase relations between cells produced therewith

163 can be very important in the construction of synthetic genetic networks and 
164 the mechanisms behind cell differentiation. Therefore, our attention will be

165 mainly devoted to clustering that occurs in the inhomogeneous states (steady

166 or oscillatory), and which could be related to biological mechanisms of dy-

167 namical differentiation. Moreover, different groupings that occur mainly in

168 the chaotic regime and contribute significantly to the complex dynamical be-

169 havior on a population-wide scale will be also of significant interest to us.

170 This is because such groupings can be also seen as a mechanism for temporal

171 mixing that enhances the diversity in the system, while mostly maintaining

172 the advantages of a synchronized (ordered collective) behavior.

173 As a first step, a minimal extension to $N=3$ identical cells was intro-

174 duced, in order to classify the dynamical changes leading to clustering. We

175 now present bifurcation diagrams for that case, with the coupling strength

$176 Q$ as the bifurcation parameter. As discussed above, $Q$ is proportional to the

177 extracellular cell density and can be changed experimentally in chemostat

178 experiments in the range between zero and one. Values beyond this range

179 do not have a biological meaning but can be helpful for the understanding

180 of the bifurcation analysis and the controlling of desired regimes. Although

181 the complete bifurcation analysis was performed using the Xppaut package

182 (Ermentrout (2002)), the diagrams presented here depict only those bifurca-

183 tion branches and points central to the corresponding discussion, in order to

184 avoid making the bifurcation charts incomprehensible.

185 The bifurcation analysis revealed a significant enlargement of the stability

186 interval for the $\operatorname{IHSS}(Q \in[0.29-0.66]$, Fig. 4(b)), in comparison to the

187 minimal case of $N=2$ coupled oscillators $(Q \in[0.36-0.55])$ (Fig. 4(a) and

188 in (Ullner et al. (2008))). The IHSS is stabilized via a Hopf bifurcation, thus 
189 displaying no qualitative changes in the mechanism of occurrence with respect

190 to the minimal model. However, the significant increase of the stability region

191 in this case ( $\approx 50 \%$ in comparison to $N=2)$ is a result of clustering, or more

192 specifically, of the increased number of possible distributions of the oscillators

193 between the two stable protein levels through which the IHSS is defined. In

194 general, given $N$ total number of cells, the oscillators can have $N-1$ different

195 distributions between the clusters (considering that the IHSS, as well as the

196 IHLC discussed below, are characterized by two-cluster decompositions).

197 In what follows, we will define the different cluster states by the notation

$198 m L \mid(N-m) U$, which denotes a cluster of $m$ oscillators in the low-protein

199 concentration state $L$, while the remaining $N-m$ oscillators populate the

200 upper state $U$, characterized by higher protein concentration. For $N=2$

201 cells, there is only one possible distribution of the oscillators in the IHSS

202 regime: $1 L \mid 1 U$ - one oscillator populates the lower, where the second one

203 populates the upper state. However, for an increased number of cells, $N=3$,

204 there are 2 different combinations, namely $1 L \mid 2 U$ (Fig. 4b, left stable branch

205 (solid (green) line)) and $2 L \mid 1 U$ (Fig. 4b, right stable branch (solid (green)

206 line)). Note that different stable cluster distributions are located on separate

207 branches of the bifurcation continuation, thus resulting in the increase of

208 the parameter interval where IHSS exists. In the case of $N=5$ oscillators,

209 for example, 4 different clusters are stable, as shown in Fig. 4(c). In that

210 diagram the cluster types are, from left to right: $1 L|4 U, 2 L| 3 U, 3 L \mid 2 U$

211 and $4 L \mid 1 U$. This particular structure of cluster distribution is typical for

212 any number of oscillators: clusters of the type $1 L \mid(N-1) U$ require small $Q$

213 values, while the $(N-1) L \mid 1 U$ exist for large $Q$. In order to understand this 
214 behavior, let us define the ratio of the oscillators distributed in the upper 215 versus lower $B_{i}$ states as $r=\frac{N-m}{m}$. Suppose that under small $Q, r$ is larger 216 than one $(r>1$ means that the majority of the oscillators in the system 217 are located in the upper $B$ state). Let us know assume that the value of $218 r$ decreases, until $r<1$. This means that the total concentration of the 219 protein $C I(B)$ in the system is decreased. Moreover, since the dynamics of 220 the $A I$ follows closely the dynamics of the protein $B$ (both expressions, that 221 of protein $B(c I)$ and of $A I$ (luxI) are regulated in a same manner via tet $R$ ), 222 the production of internal $A I$ will also be decreased. In order to compensate 223 for the lack of internal $A I$ which will destabilze the $I H S S$, the re-influx of 224 external $A I$ needs to be increased. This will subsequently lead to higher $Q$ 225 values which means that in general, if $r<1$, larger $Q$ is necessary to observe 226 stable IHSS distributions.

227 The left and middle plots in Fig. 5 show time traces for two different clus228 ter decomposition in the $I H S S$ regime for $N=18$. Each possible partition 229 shows slightly different levels in the protein concentrations, and hence a fine 230 tuning of the protein levels can be accomplished by choosing a specific $Q$ in231 terval for proper partition of the oscillators. This specific effect enhances, on 232 the one hand, the biotechnological applications of synthetic genetic networks 233 by providing a possible method for fine manipulation of the protein concen234 tration level, and on the other hand it might be seen as typical adaptability 235 of a cell population: when changes in the environmental conditions occur, 236 the population can easily adjust its cell distribution, adapting optimally to 237 the environment.

238 The Hopf bifurcation through which the IHSS is stabilized gives rise also 
239 to a branch of stable inhomogeneous limit cycles $(I H L C)$, already introduced

240 for the case of $N=2$ oscillators in Fig. 2(d) (the corresponding bifurcation

241 diagram is given in Fig. 6(a)). However, in comparison to that minimal case,

242 the IHLC regime for higher population sizes is more complex, due to the

243 increased number of possibilities for distributing the oscillators in the two

244 clusters (a stable and an oscillating one).

245 Analogous to the formation of $I H S S$ clusters for $N=3$, two distinct

246 IH LC clusters can be observed here as well. We have identified these regimes

247 as $1 L \mid 2 U$ (left solid (red) line in Fig. 6, (b)), and $2 L \mid 1 U$ (right solid (red)

248 line in Fig. 6 (b)), emerging from the correspondent IHSS branches (Hopf

249 bifurcations of the $I H S S, H B_{s 1}$ and $H B_{s 2}$ in Figs. 4(b), 6(b)). This results

250 again in an interval where the $I H L C$ regime is stable in comparison to the

251 2-oscillator case (compare Figs. 6 (a) and (b)). Moreover, the IHSS and

$252 I H L C$ regimes coexist in certain $Q$ ranges (for instance, Figs. 5 and 6). This

253 new behavior is also a result of the formation of clusters for increasing system

254 size. The possibility that one of the $I H L C$ distributions will overlap with the

255 I HSS from another cluster distribution (containing less elements in the lower

256 level) increases significantly. Hence, we can state that the increase of the

257 stability regions of the inhomogeneous states (IHSS and IHLC) unraveled

258 by the numerical simulations (Fig. 3) is a result of the cluster formation for

259 growing populations, as we have determined from the bifurcation analysis 260 presented.

261 Interestingly, the population displays even more complicated behavior 262 when analysing the clustering effect in the $I H L C$ regime. This complexity

263 is manifested through the formation of sub-clusters in the lower (oscillatory) 
264 state, where oscillators exhibit identical behavior within a single sub-cluster,

265 but with various phase relations among them. The simplest case consists of 266 only two oscillators located on the lower oscillatory level - they are organized

267 in anti-phase. However, increasing the number of oscillators in the lower state

268 reveals multitude of relations between the oscillators grouped in sub-clusters

269 and hence, besides the distribution of oscillators between the upper and lower

270 states, one needs to consider also the composition of the oscillatory sub-

271 clusters in the lower protein level. Figures 7 to 9 illustrate some examples of

272 possible combinations of partitions and phase relations in the sub-threshold

273 oscillations for an ensemble of $N=18$ oscillators which we discuss next.

274 Due to the technical difficulty to handle a high-dimensional system with

275 the Xppaut package, we present here only numerical findings. Please note

276 the non-uniform distribution of the phase in some situations. The basic

277 distribution of IHLC can be seen in Fig. 7(left), with only one element in

278 the lower state. However, if an additional element is located in the lower

279 state as well, (Fig. 7, middle), the phase-repulsive cell-to-cell communication

280 evokes anti-phase oscillations, as already mentioned. In the situation where

281 three cells are located in the lower state, stable out-of-phase oscillations with

282 a phase-shift of $\frac{2 \pi}{3}$ between clusters (Fig. 7, right) are observed, since the

283 phase-repulsive coupling maximizes their phase difference.

284 Additional oscillators in the lower state contribute to the formation of 285 more complex distributions. A fourth cell expressing a low $C I$ protein con286 centration can establish a separate sub-cluster, which leads to the regime 287 (1:1:1:1)L $\mid 14 U$ (each sub-cluster is composed of isolated cells, as shown 288 in Figs. 8, left and middle) or to a sub-cluster with more than one cell (e.g. 
289 two sub-clusters are formed, each containing two cells, as in Fig. 9 middle).

290 It is interesting to note, that the regime $(1: 1: 1: 1) L \mid 14 U$ has two re-

291 alizations with different phase relations between the self-oscillatory cells in

292 the low $C I$ level. The left panel of Fig. 8 shows a nearly equal spaced phase

293 shift of about $\frac{2 \pi}{4}$, while the middle panel shows the case of an inhomogeneous

294 phase shift. In particular, the cells are observed to come close to each other

295 (two of them), but they never merge to form a sub-cluster. The right panel

296 in Fig. 8 shows a more complex phase relation in which 5 out of the 18 cells

297 are in the low-protein state, oscillating separately with an inhomogeneous

298 phase-difference. This phase-regime is similar to the one in the middle plot

299 of Fig. 8, but with an additional oscillator in between the other four clusters.

300 The corresponding six time series given in Figs. 7-8 correspond to the same

301 parameter values, for $N=18$ and a fixed coupling value $Q=0.2$. Thus

302 the different dynamical behavior observed here originates only from different

303 initial conditions.

304 It is important to note once again that a particular cluster distribution

305 is characterized with a distinct level of protein concentration expressed in

306 the cell. Namely, larger number of oscillators in the lower state reduces the

307 protein concentration in the higher state. Moreover, we have also observed

308 that the ratio $\left(r=\frac{N-m}{m}\right)$ of the number of oscillators in both levels affects

309 the amplitude of the limit cycle oscillations located in the lower protein level:

$310 r<1$ results in increased amplitude values. Additionally, the ratio $r$ con-

311 tributes to the changes in the period of oscillation, having as a consequence

312 a well pronounced multirhythmicity in the system. Table 1 lists the observed

313 periods and phase relations for the different ratios in the cases discussed in 
314 Figs. 7-8. In the present case of $N=18$ and $Q=0.2$, every additional 315 oscillator in the lower $C I$ level of the IHLC lengthens the period by $\approx 1.2$ 316 time units, which leads to a significant change in the period between different 317 distributions. A modification of the phase relation by a fixed partition ratio 318 does not influence the period (left and middle panels of Fig. 8).

319 Due to the dynamical complexity of the system, which as mentioned 320 above is a direct consequence of the clustering, it is useful to look into the 321 stability of the different regimes (HSS, IHSS, IHLC), in order to define 322 a general prediction scheme to determine which solution is dominant under 323 different conditions. We have therefore calculated 1000 time series for a 324 growing population size $N$, with different random initial conditions for every 325 parameter set, using the approach discussed in Sec. 2. These initial conditions 326 cover the $7 N$-dimensional phase space of the system ( 7 degrees of freedom 327 per oscillator) densely enough such that one can detect stable coexisting 328 attractors with a significant basin of attraction. Figure 10 shows in detail the 329 system size effect for two specific coupling values, $(Q=0.24$ and $Q=0.3)$.

330 In both cases, the results show a clearly monotonic increase of the probability 331 that the IHSS is reached from random initial conditions at the expense of the $332 H S S$, as the size of the population grows. For ensembles larger than several 333 hundred cells the IHSS is the dominant region, allowing us to speculate that 334 the artificial differentiation of cells is strongly dependent on the size of the 335 population, becoming more likely with proliferation (Koseska et al. (2009)). 336 In other words, the stable inhomogeneous steady state resembles Turing's 337 dissipative structure (Turing (1952)), only without space variables. In a 338 sense, instead of the spatial Turing structure, in IHSS we have a two cluster 
339 decomposition present. This state, as discussed above, is characterized by

340 two different stable protein concentration levels, which might be biologically

341 interpreted as dynamical differentiation. Thus, one can further speculate

342 that a robust dynamical differentiation of the cells strongly depends on the

343 size of the population.

344 4. Full-amplitude oscillatory regimes - Regular and chaotic attrac345 tors

346 For couplings smaller than a given critical value $Q_{\text {crit }} \sim 0.129$ (the value 347 of $Q_{\text {crit }}$ slightly varies depending on $N$ ), the system can only exhibit self348 oscillatory solutions: we have identified out-of-phase oscillations with a num349 ber of different phase-shifts (e.g. $\frac{\pi}{2}, \frac{3 \pi}{4}$, etc.). In contrast to the $I H L C$ so350 lution, the attractors here share the same phase space. These full-amplitude 351 oscillatory regimes, as we will further denote them, are dynamically very 352 rich, displaying a diversity of sub-regimes for increasing coupling values. In 353 particular, similarly to the minimal system of $N=2$, we found two main 354 types of behavior depending on the coupling strength $Q$ :

355 - regular oscillations with stable cluster formation;

356 - chaotic self-oscillations with only a temporary cluster formation (we 357 will denote this as grouping in the following discussion).

358 In what follows, we investigate the general characteristics of these regimes 359 by means of bifurcation and numerical analysis. However, in order to dis360 tinguish between separate cluster formations for increasing population sizes 361 via numerical simulations, we use the following definition: oscillators $i$ and 
$362 j$ belong to the same cluster $K$ at time $t$ if the difference between the inter363 nal $A I$ concentrations $S_{i}(t)$ and $S_{j}(t)$ at consecutive sampling time events is 364 smaller than a pre-defined value $\varepsilon=0.001$ :

$$
\begin{aligned}
& {\left[\operatorname{osc}_{i}(t), \operatorname{osc}_{j}(t)\right] \in \operatorname{clust}_{K}(t)} \\
& \text { if }\left|S_{i}(t)-S_{j}(t)\right| \leq \varepsilon \text { and } \\
& \left|S_{i}(t-\Delta t)-S_{j}(t-\Delta t)\right| \leq \varepsilon
\end{aligned}
$$

365 The cluster sampling occurs every $\Delta t=64$ time units, which is larger than

366 the average period of the oscillations (the average period of oscillations is

367 between 40 and 50 time units or approx. $200 \mathrm{~min}$ ). Using these criteria, we

368 classify the clustering of the oscillators, and present the resulting structures

369 in the form of cluster plots (Figs. 12, 13, 15, 16), where the $x$-axes denote

370 time, and the $y$-axes represent the oscillator index. There, we use different

371 colors to encode the cluster number to which each element belongs (white

372 color represents a free-running oscillator, which does not belong to any of

373 the clusters). Although these plots do not show the dynamics of the self-

374 oscillations in detail, they focus on the difference in the protein concentrations

375 of separate cells over time, and therefore enable a visualization of the long-

376 time cluster dynamics.

377 4.1. Regular attractors

378 Under small and intermediate couplings $(0 \leq Q \lesssim 0.55)$, the system 379 demonstrates regular oscillations with a fixed unique amplitude and com380 mon period for all oscillators (Fig. 12, top panel). In this case, the repulsive 381 cell-to-cell communication evokes the preference of phase-shifted oscillations, 
382 and small ensembles with $N \leq 4$ show solutions without clustering and ho383 mogeneously distributed phases (Fig. 2c). The phase shift here depends on 384 the size of the system, and obeys the relation $\frac{2 \pi}{N}$ (a solution known also 385 as a "splay-phase" solution (Kaneko (1991); Watanabe and Strogatz (1993); 386 Nicolis and Wiesenfeld (1992))). Hence, in a system of three coupled repres387 silators, one can find stable full-amplitude oscillations phase-shifted by $\frac{2 \pi}{3}$ 388 between $\operatorname{HB}_{1}(Q=1.253)$ and $\mathrm{TR}_{1}$ (torus bifurcation for $Q=1.11$ ), and 389 from $Q=0$ until $\mathrm{TR}_{2}=0.55$ (see Fig. 11). No other stable cluster decom390 position was identified from the bifurcation analysis. For $Q<0.129$, and as 391 mentioned above, this is the only stable solution of the system, whereas for 392 increasing coupling the full-amplitude regime coexists with HSS, IHSS, and 393 IHLC states, as shown in Fig. 11.

394 In systems where $N>5$, clustering is observed in the regular oscillatory 395 regime. Here, the 3-cluster decomposition dominates, with a nearly equal 396 number of oscillators in each one (for details see Table 2), and a distinct phase 397 relation between separate clusters. Morever, the periods of oscillation are 398 slightly shorter than those of isolated elements, and decrease as $Q$ increases 399 (Table 2). We show here an example of a system of $N=18$ cells. The 400 onset of clustering can be seen in the cluster plot in the bottom panel of 401 Fig. 12. After a long transient (about 1.200 time units, in this particular 402 case) a distribution of three clusters is stabilized, with a $7: 6: 5$ distribution 403 of oscillators (cells) between the clusters, and a phase shift of about $\sim \frac{2 \pi}{3}$ 404 among them. Time series of the separate clusters (the oscillators within 405 each cluster display synchronous behavior) are given in the top panel of 406 Fig. 12 (the coloring coresponds to the cluster plot). The long transient in 
407 the simulation looks unphysiological at first glance, but all simulations are 408 drawn from random initial conditions with a very large diversity amongst 409 the cells. We use those unrealistic initial conditions in order to underline 410 the ability of the system to form stable clusters under any condition. After 411 proliferation, the daughter cells are in a similar phase as the mother, which 412 decreases the formation of stable clusters significantly.

413 In the cases investigated, all the oscillators are identical and coupled via a 414 mean field, hence there are no preferences amongst them to establish a given 415 set of clusters. Thus the distribution of the oscillators between the clusters 416 depends exclusively on the initial conditions. Several typical attractors ob417 served for different system sizes and coupling values $Q$ are listed in Table 4182 , together with the values of their periods. As shown here, the 3-cluster 419 decomposition with nearly equal distribution of oscillators between the clus420 ters dominates for large system sizes and over wide ranges of coupling. An 421 exception to this case, as discussed above, is the formation of clusters for $422 N \leq 4$. In the case for $N=4$, for instance, the 3-cluster decompositions 423 lose stability, and stable 4-cluster decompositions are formed. Interestingly, 424 the coupling $Q$ has an inverse influence on the oscillation period. Normally, 425 stronger coupling lengthens the period of coupled systems (Crowley and Ep426 stein (1989); Volkov and Stolyarov (1994)), but the situation is different in 427 the present case because $Q$ controls the reinflux of AI and a higher internal 428 AI concentration shortens the repressilator cycle. Compared to the coupling 429 strength $Q$, the system size $N$ and the cluster composition have a minor 430 influence on the period. 
431

432

433

434

435

436

437

438

439

440

441

442 is realistic, since this parameter defines the expression strength of the re-

443 pressilator genes, which is proportional to the concentration of repressilator

444 plasmids present in the cell. The control of the number of plasmid copies in

445 experiments was discussed in (Paulsson and Ehrenberg (2001)), and it can

446 be coordinated with the cell's growth and division. Additionally, an increase

447 in $\alpha$ lengthens the period of oscillations, as already mentioned.

448 We have observed that even in the presence of diversity, the three-cluster 449 decomposition is the most probable state in the system (an example for $450 Q=0.5$ is given in Fig. 13). However, in contrast to the case of identical 451 oscillators, some of the cells (in the case of Fig. 13, the two elements with 452 the smallest parameter $\alpha$, i.e. the cells with the shortest period), are not 453 phase locked and jump periodically from one of the three stable clusters to 454 the other one. Moreover, the heterogeneity introduced via the parameter 455 mismatch breaks the symmetry present in the system of identical oscillators, 
456 and leads to a situation where oscillators with similar properties (i.e. similar $\left.457 \alpha_{i}\right)$ group together in a cluster, preferentially.

\section{4.3. Irregular self-oscillations}

459 The bifurcation analysis we have performed on the model for $N=3$ cells 460 shows that for $Q \gtrsim 0.55$, the system goes beyond the range of regular oscil461 lations: the periodic branch loses its stability between two torus bifurcations 462 ( $\mathrm{TR}_{1}$ and $\mathrm{TR}_{2}$ in Fig. 11 ), which contributes to the appearance of oscilla463 tions with strong variations of the amplitude. These irregular oscillations 464 look very similar to chaotic time series (Fig. 15, top plot), however, in order 465 to classify this as a chaotic behavior, certain criteria need to be fullfilled, 466 e.g. at least one of the Lyapunov exponents of the system needs to be pos467 itive. Therefore, we integrate forward in time a small perturbation of the 468 trajectory, the random tangent vector, by means of the Jacobi matrix. The 469 logarithm of the norm of the tangent vector is related to the maximal Lya470 punov exponent (Eckmann and Ruelle (1985)) and we normalize it by the 471 integration time. The result is plotted in the top panel of Fig. 14, and shows 472 that for $Q>Q_{\text {chaos }} \approx 0.6$ (upper boundary of $Q \approx 1$ ), clear chaotic behavior 473 with a positive maximal Lyapunov exponent is observed. The bottom panel 474 of Fig. 14 displays a bifurcation diagram computed as a series of Poincaré 475 sections, with the ordinate showing the value of the $B_{1}$ when the trajectory 476 crosses $A_{1}=4.0$. We avoid the tracking and evaluation of unstable attractors 477 and numerical artifacts by adding small dynamical noise to the transcription 478 dynamics of the tetR $m R N A$, i.e. we add the term $\xi_{i}(t)$ to the rhs of Eq. 1. 479 The local noise term $\xi_{i}(t)$ is assumed to be Gaussian, with zero mean and 480 intensity $\sigma_{a}^{2}$ defined by the correlation $\left\langle\xi_{i}(t) \xi_{j}(t+\tau)\right\rangle=\sigma_{a}^{2} \delta(\tau) \delta_{i, j}$. Beside 
481 being technically useful, the noise is biologically relevant, as it is caused by 482 random fluctuations in transcription due to the small number of involved $483 m R N A$ (Elowitz and Leibler (2000)).

484 In contrast to the case of the minimal system $(N=2)$, where a similar 485 irregular dynamic with chaotic behavior was observed (Ullner et al. (2008)), 486 the extended system studied here $(N=18$ in Fig. 14) is characterized by 487 the ability to build temporal clusters, which we refer to as grouping. The 488 coupling $Q$ changes the chaotic behavior gradually (top panel in Fig. 14). 489 A first weak increase of the maximal Lyapunov exponent is followed by a 490 fast rising interrupted by short collapse and a final decline to zero at $Q \approx 1$. 491 The degree of chaos influences the grouping ability, as chaos destabilizes and 492 shortens the grouping. In the parameter range of $Q$ where more irregular than 493 simple periodic oscillations are observed, $0.55 \lesssim Q$, temporal $2-, 3-, 4-$ or 4945 - grouping decompositions with a significant lifetime have been observed, 495 in contrast to the regular attractors, where the 3-cluster decompositions 496 were the dominant ones.

497 The example in Fig. 15 shows a weak chaotic dynamics of $N=18$ os498 cillators at $Q=0.6$, with long-living 3 - and 4-grouping constellations. The 499 cluster plot (bottom panel of the figure) illustrates the interplay of long-time 500 grouping and recurring transients with less ordered states, while a rearrange501 ment to a new grouping happens. The groupings are long living up to 20, 000 502 time units, i.e. about 4000 cycles. Due to the symmetry of the system in 503 the case of identical elements, separate oscillators do not have local group504 ing preferences. The bottom plot of Fig. 15 gives a detailed insight on the 505 oscillatory dynamics at different times, and shows the long-living 3- and 4- 
506 grouping constellations, and transients with a high degree of decomposition.

507 However, once the oscillators are distributed in a long-living grouping state,

508 they oscillate synchronously within the group and cannot be distinguished by

509 their time series until the next decomposition occurs and spreads the phases.

510 The second example of irregular chaotic self-oscillations (Fig. 16) illus-

511 trates a regime of fully developed chaos at high coupling $Q=0.75$. The max-

512 imal Lyapunov exponent (Fig. 14) increases significantly above zero, which

513 confirms the chaotic character of the dynamics. Interestingly, the temporal

514 grouping of the oscillators is conserved, but with a significantly shorter life-

515 time and faster mixing as compared to the weak chaotic dynamics discussed

516 above (Fig. 15). Despite the fact that clusters are not stable in this param-

517 eter range, some of the oscillators run in-phase over long time and fulfill the

518 clustering condition (Eq. 10) temporarily. In this typical situation shown

519 here for fully developed chaos at $Q=0.75$ (Fig. 16) the grouping of the

520 oscillators can last over a relatively long time and cover up to 5,000 time

521 units, i.e. more than 100 oscillations. The individual repressilators oscillate

522 in an irregular manner with fluctuating amplitude and period. The top pan-

523 els of Fig. 16 shows different snapshots of the time series, and the bottom

524 panel of the figure shows the corresponding cluster plot and illustrates the

525 interplay between grouping and decomposition. Note the larger maximal am-

526 plitudes in the more ordered case than in the situation where higher degree

527 of decomposition is observed.

528 In general, it can be stated that inside the chaotic ensemble there exists

529 an everlasting tendency to build and break temporal groups, which leads to

530 their mixing. Many different temporal distributions of the oscillators into 
531 groups are possible, which survive over several oscillation periods until the 532 next mixing occurs. In the case investigated here in detail, $N=18$ oscilla533 tors, we have observed e.g. two temporal groups with a distribution $9: 9$ of 534 the oscillators between them, three groups with distributions $7: 6: 5$, and $5357: 7: 4$, four temporal groups with distributions $8: 6: 3: 1,7: 6: 4: 1$, $5365: 5: 5: 3$, and $5: 5: 4: 4$, as well as several examples of five groupings 537 decompositions, with distributions such as $6: 5: 4: 2: 1,5: 5: 4: 2: 2$, $5385: 5: 4: 3: 1$ or $5: 4: 4: 3: 2$. Several other examples with a 539 higher degree of decomposition can be observed as well, however, they ex540 hibit a much shorter lifetime. The observed clustering effects resemble the 541 dynamical behavior of globaly coupled maps reported by Kaneko in (Kaneko 542 (1990)). The transition from an ordered to a partially ordered and turbulent 543 phase, where the number of clusters is significantly increased is similar to 544 the case of a weak and well developed chaotic clustering decomposition or545 dering, discussed in this section. Moreover, we show that a growing system 546 size increases the possibility for grouping formation significantly, as well as 547 the number of different distributions of the oscillators between the groups, 548 which on the other hand enhances the flexibility of the system. This means 549 that by varying environmental conditions, the population can switch between 550 different distributions to adapt to its surroundings and improve its fitness.

551 Although the chaotic dynamics observed here and the effect of intrinsic noise 552 in synthetic oscillators (Elowitz and Leibler (2000); Stricker et al. (2008)) 553 have very similar manifestations despite their different origins, we intend to 554 draw the readers' attention to chaos as an alternative source of uncertainty 555 in genetic networks. 
556 The chaotic dynamics and the grouping phenomena appear graduately 557 for increasing coupling $Q$, i.e. at cell densities which could be a cause for

558 stress. One could speculate, that the population has the flexibility to respond 559 to environmental stress by distributing its cells within stable clusters, and 560 thus increasing the variability and diversity amongst the different cells to 561 enhance the probability to survive the stress situation. The gradual chaotic 562 behavior enables the population to adapt the mixing velocity and the degree 563 of diversity to the stress conditions.

\section{5. Discussion}

565 The mechanism how a multicellular system assures a robust and coordi566 nated response in a noisy and fluctuating environment is still an intriguing 567 question. It has been suggested however, that the intercellular signaling plays 568 one of the crucial roles in the establishment of cooperative functioning in 569 populations. In that contex, we attempt here to characterize the dynamical 570 behavior of multicellular systems using phase-repulsively coupled synthetic 571 genetic repressilators [Eqs. (1)-(7)]. The focus in the current paper is on the 572 dynamics of large and growing ensembles, but we also compare our results 573 with the recent findings on the basic ensemble of two coupled repressilators, 574 by means of numerical simulations of the dynamics and bifurcation analysis. 575 We show that a multicellular population of synthetic genetic repressila576 tors displays various dynamical behavior, e.g. full-amplitude self-oscillations, 577 homogeneous steady state (HSS), inhomogeneous steady state (IHSS), and 578 inhomogeneous limit cycle $(I H L C)$. These regimes are present for all popu579 lation sizes, and may in general coexist with each other. Moreover, the size of 
580 the system affects the relative sizes of the basins of attraction of each regime.

581 For instance, the inhomogeneous states become more likely for larger popu-

582 lations. Interestingly, those inhomogeneous regimes can be associated with

583 permanent artificial cell differentiation in synthetic genetic networks, and

584 the simulations predict that a growing system size, e.g. due to proliferation,

585 enhances the probability of differentiation. Additionally, large system sizes

586 widen the parameter range of the $I H L C$ and IHSS regimes significantly,

587 and further enhance the differentiation probability.

588 Furthermore, the understanding of cell differentiation (Kaneko and Yomo

589 (1997); Furusawa and Kaneko (2001)) and its connection to the emergence

590 of stable attractors from cell-to-cell coupling is still not clear. Here, we

591 have investigated systematically the mechanisms through which coexisting

592 attractors arise in the multicellular system. Namely, a closer look into the

593 inhomogeneous regimes ( $I H L C$ and $I H S S$ ) shows a splitting of the single at-

594 tractor that exists for two coupled elements into multiple coexisting solutions

595 for many oscillators, and the number of stable attractors increases with the

596 system size. A combination of numerical simulations and bifurcation analysis

597 revealed that the different stable IHSS solution branches differ by the num-

598 ber of elements in the high and low protein levels, and that each distribution

599 implies a new attractor with different stability ranges and individual protein

600 levels. The IHLC is on the other hand, directly bounded to the IHSS with

601 the same distribution of the oscillators via a Hopf bifurcation. The ability of

602 the $I H L C$ regime to build oscillating clusters with different phase relations

603 in the low protein level increases further the number of sub-regimes, and in-

604 cludes multi-rhythmicity as a tunable clock (similarly to the tunability of the 
605 IHSS regimes) because the attractors express different typical frequencies. 606 One can further speculate that this feature can be seen as an example of 607 the adaptability of a population to easily adjust its cell distribution when 608 environmental changes occur, in order to respond and adapt optimaly to the 609 surrounding.

610 Similar behavior was observed in the full-amplitude self-oscillatory regime: 611 various oscillatory clusters with different partitions of the oscillators amongst 612 them. Moreover, the spectra of possible constellations increases with the sys613 tem size. For small and intermediate coupling $Q$, stable self-oscillations char614 acterized with a 3 -cluster decomposition, and phase shifted by $\sim \frac{2 \pi}{3}$ appear. 615 These 3-cluster decompositions are very robust to perturbations, in the form 616 of e.g. random initial conditions, dynamical noise or parameter heterogene617 ity. The direct numerical investigations performed here can not guarantee 618 the "mathematical" stability of the discussed clustering regimes. However, 619 taking in mind recent results (Ashwin et al. (2007)) we can suggest that these 620 clusters belong to a heteroclinic network and demonstrate switching which is 621 manifested more effectively in the presence of detuning. This question itself 622 deserves further attention but in any case, the regimes observed here have 623 very long life times which certainly makes them interesting and important 624 for biology.

625 Furthermore, an increasing cell density caused by cell growth and pro626 liferation increases effectively the coupling $Q$, and up to a critical coupling $627 Q_{\text {crit }} \approx 0.6$ the regular self-oscillations become unstable, turning into chaotic 628 oscillations with high variability in their amplitude and frequency. Interest629 ingly, also in the presence of chaos the population tends to build temporal 
630 clusters, which we refer to as groups. These temporal groups of co-jointly 631 oscillating repressilators have a significant lifetime, depending on the degree 632 of chaos, and are interrupted by recurring decomposition of the groups and 633 a reassembling into different groups. The chaotic dynamics appears gradu634 ally with $Q$, and allows the population to respond flexibly and sensitively to 635 increasing stress via a higher dynamical diversity inside the ensemble.

636 In summary, our results show that a population of synthetic genetic clocks 637 coupled via the mean field exhibit a significantly enhanced range of possi638 ble dynamical regimes with very different properties. One could speculate 639 that the observed multi-stability and multi-rhythmicity, which increase with 640 the system size, enhance the fitness of the cellular population under envi641 ronmental stress, and optimize the adaptation of the colony by a sensitive 642 adjustment of the protein dynamics.

\section{6. Acknowledgments}

644 A.K. and J.K. acknowledge the GoFORSYS project funded by the Fed645 eral Ministry of Education and Research, Grant Nr. 0313924, E.U. acknowl646 edges financial support from SULSA, Deutsche Forschungsgemeinschaft (SFB

647 618) and the Alexander von Humboldt Foundation, E.V. the Program "Ra648 diofizika" Russian Academy and RFBR Grant No. 08-0200682, 08-0100131, 649 J.G.O. the Ministerio de Ciencia e Innovacion (Spain) (project FIS2009-13360 650 and I3 program) and J.K. the EU through the Network of Excellence BioSim, 651 Contract No. LSHB-CT-2004-005137. This work has also been supported by 652 the European Commission (project GABA, FP6-NEST contract 043309). 


\section{References}

654 Ashwin, P., Orosz, G., Wordsworth, J., Townley, S., 2007. Dynamics on 655 networks of cluster states for globally coupled phase oscillators. SIAM J. 656 Appl. Dyn. Syst. 6, 728-758.

657 Balagadde, F. K., Song, H., Collins, C. H., Barnet, M., Arnold, F. H., Quake, 658 S. R., You, L., 2008. A synthetic escherichia coli predator-prey ecosystem. 659 Mol. Sys. Bio. 4:187, 1-8.

660 Crowley, M. F., Epstein, I. R., 1989. Experimental and theoretical studies 661 of a coupled chemical oscillator: phase death, multistability and in-phase 662 and out-of-phase entrainment. J. Phys. Chem. 93, 2496-2502.

663 Eckmann, J. P., Ruelle, D., 1985. Ergodic theory of chaos and strange at664 tractors. Rev. Mod. Phys. 57 (3), 617-656.

665 Elowitz, M., Leibler, S., 2000. A synthetic oscillatory network of transcrip666 tional regulators. Nature 403, 335-338.

667 Ermentrout, B., 2002. Simulating, Analyzing, and Animating Dynamical Sys668 tems: A Guide to Xppaut for Researchers and Students (Software, Envi669 ronments, Tools). SIAM Press.

670 Furusawa, C., Kaneko, K., 2001. Theory of robustness of irreversible dif671 ferentiation in a stem cell system: chaos hypothesis. J. theor. Biol. 209, $672 \quad 395-416$.

673 García-Ojalvo, J., Elowitz, M. B., Strogatz, S. H., 2004. Modeling a synthetic 
674

675

676 Golomb, D., Hansel, D., Shraiman, B., Sompolinsky, H., 1992. Clustering in

677 globally coupled phase oscillators. Phys. Rev. A 45, 3516-3530.

678 Heinlein, M., 2002. Plasmodesmata: dynamic regulation and role in macro679 molecular cell-to-cell signaling. Curr. Opin. Plant Biol. 5, 543-552.

680 Kaneko, K., 1990. Clustering, coding, switching, hierarchical ordering, and 681 control in a network of chaotic elements. Physica D 41, 137-172.

682 Kaneko, K., 1991. Globally coupled circle maps. Physica D 54, 5-19.

683 Kaneko, K., Yomo, T., 1994. Cell division, differentiation and dynamic clus684 tering. Physica D 75, 89-102.

685 Kaneko, K., Yomo, T., 1997. Isologous diversification: A theory of cell dif686 ferentiation. Bull Math Biol. 59, 139-196.

687 Kiss, I. Z., Hudson, J. L., 2003. Chaotic cluster itinerancy and hierarchical 688 cluster trees in electrochemical experiments. Chaos 13, 999-1009.

689 Koseska, A., Volkov, E., Zaikin, A., Kurths, J., 2007. Inherent multistability 690 in arrays of autoinducer coupled genetic oscillators. Phys. Rev. E 75 (3), $691031916(8)$.

692 Koseska, A., Zaikin, A., Kurths, J., García-Ojalvo, J., 2009. Timing cellular 693 decision making under noise via cell-cell communication. PLoS ONE 4, $694 \quad \mathrm{e} 4872$. 
695 Kuznetsov, A., Kærn, M., Kopell, N., 2004. Synchrony in a population of 696 hysteresis-based genetic oscillators. SIAM Journal on Applied Mathematics $697 \quad 65(2), 392-425$.

698 Kuznetsov, A., Kurths, J., 2002. Stable heteroclinic cycles for ensembles of 699 chaotic oscillators. Phys. Rev. E 66, 026201.

700 Manruiba, S., Mikhailov, A., 1999. Mutual synchronization and clustering 701 in randomly coupled chaotic dynamical networks. Phys. Rev. E 60, 1579$702 \quad 1589$.

703 McMillen, D., Kopell, N., Hasty, J., Collins, J. J., 2002. Synchronizing genetic 704 relaxation oscillators by intercell signaling. Proc. Natl. Acad. Sci. U.S.A. $70599(2), 679-684$.

706 Miyakawa, K., Yamada, K., 2001. Synchronization and clustering in globally 707 coupled salt-water oscillators. Physica D 151, 217-227.

708 Nakajima, A., Kaneko, K., 2008. Regulative differentiation as bifurcation of 709 interacting cell population. J Theor. Biol. 253(4), 779-787.

710 Nicolis, S., Wiesenfeld, K., 1992. Ubiquitous neutral stability of splay-phase 711 states. Phys. Rev. A 45, 8430-8435.

712 Okuda, K., 1993. Variety and generality of clustering in globally coupled 713 oscillators. Physica D 63, 424-436.

714 Osipov, G., Kurths, J., Zhou, C., 2007. Synchronization in oscillatory net715 works, 1st Edition. Springer, Berlin. 
716 Paulsson, J., Ehrenberg, M., 2001. Noise in a minimal regulatory network: 717 plasmid copy number control. Q. Rev. Biophys. 34, 1-59.

718 Perbal, B., 2003. Communication is the key. Cell Commun. Signaling 1, 1-4.

719 Stricker, J., Cookson, S., Bennett, M. R., Mather, W. H., Tsimring, L. S., 720 Hasty, J., 2008. A fast, robust and tunable synthetic gene oscillator. Nature $721 \quad 456,516-519$.

722 Taga, M. E., Bassler, B. L., 2003. Chemical communication among bacteria. 723 Proc. Natl. Acad. Sci. U.S.A. 100, 14549-14554.

724 Tanouchi, Y., Tu, D., Kim, J., You, L., 2008. Noise reduction by diffu725 sional dissipation in a minimal quorum sensing motif. PLoS Comp. Biol. $726 \quad 4, \mathrm{e} 1000167$.

727 Turing, A., 1952. The chemical basis of morphogenesis. Phil. Trans. R. Soc. 728 Lond. B 237, 37-72.

729 Ullner, E., Koseska, A., Kurths, J., Volkov, E., Kantz, H., García-Ojalvo, J., 730 2008. Multistability of synthetic genetic networks with repressive cell-to731 cell communication. Phys. Rev. E 78, 031904.

732 Ullner, E., Zaikin, A., Volkov, E. I., García-Ojalvo, J., 2007. Multistabil733 ity and clustering in a population of cellular genetic oscillators via phase 734 repulsive cell-to-cell communication. Phys. Rev. Lett. 99, 148103.

735 Volkov, E. I., Stolyarov, M. N., 1994. Temporal variability in a system of 736 coupled mitotic timers. Biol. Cybern. 71, 451-459. 
737 Wang, W., Kiss, I. Z., Hudson, J. L., 2001. Clustering of arrays of chaotic 738 chemical oscillators by feedback and forcing. Phys. Rev. Lett. 86, 49547394957.

740 Watanabe, S., Strogatz, S. H., 1993. Integrability of globally coupled oscilla741 tor array. Phys. Rev. Lett. 70, 2391-2395.

742 You, L., Cox III, R. S., Weiss, R., Arnold, F. H., 2004. Programmed popula743 tion control by cell-cell communication and regulated killing. Nature 428, $744 \quad 868-871$.

745 Zhou, T., Zhang, J., Yuan, Z., Chen, L., 2008. Synchronization of genetic $746 \quad$ oscillators. Chaos 18, 037126. 


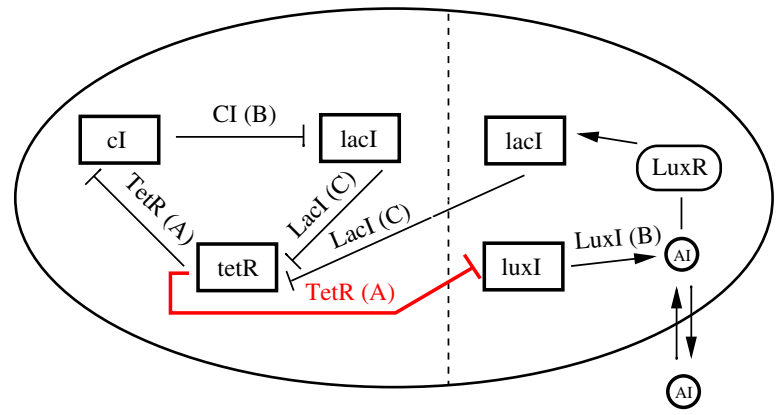

Figure 1: 

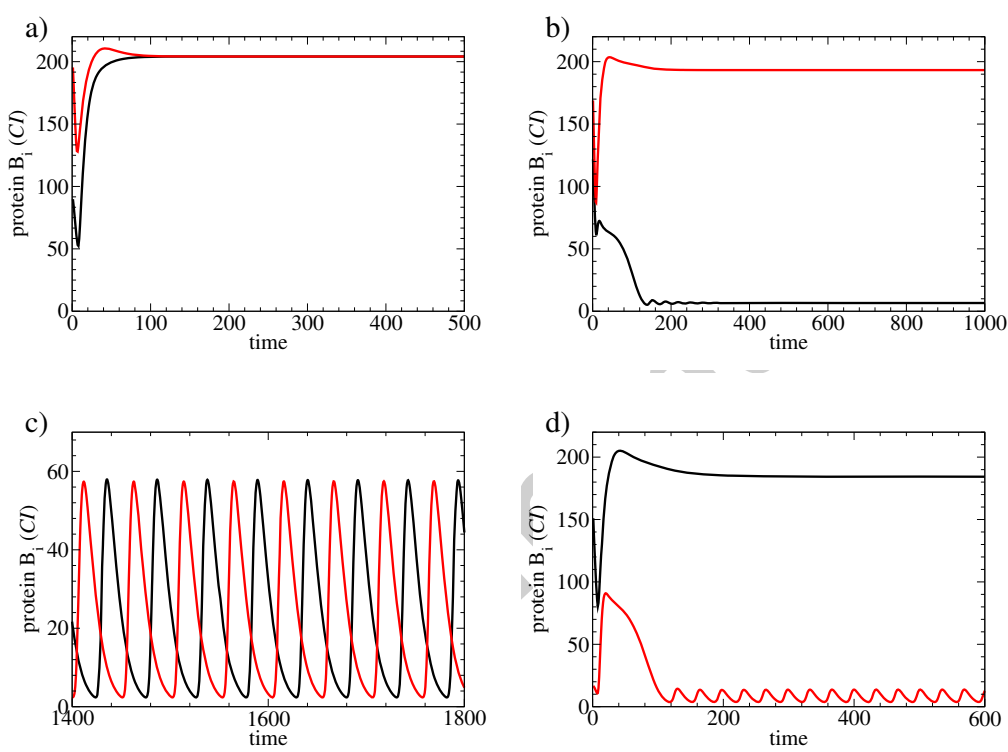

Figure 2: 


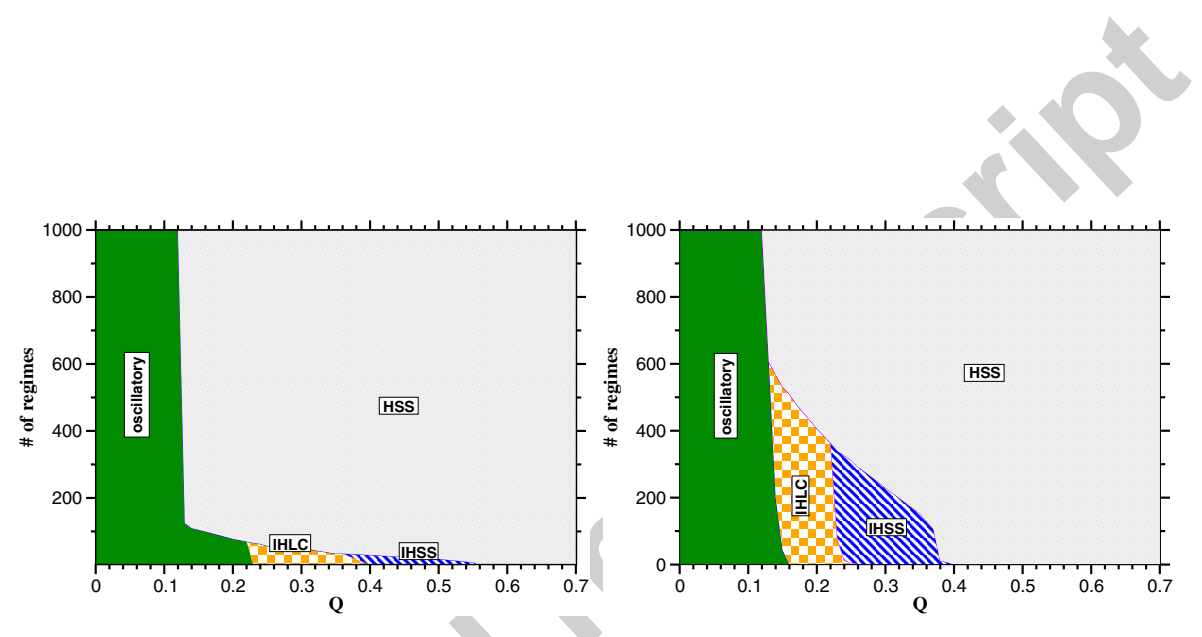

Figure 3: 

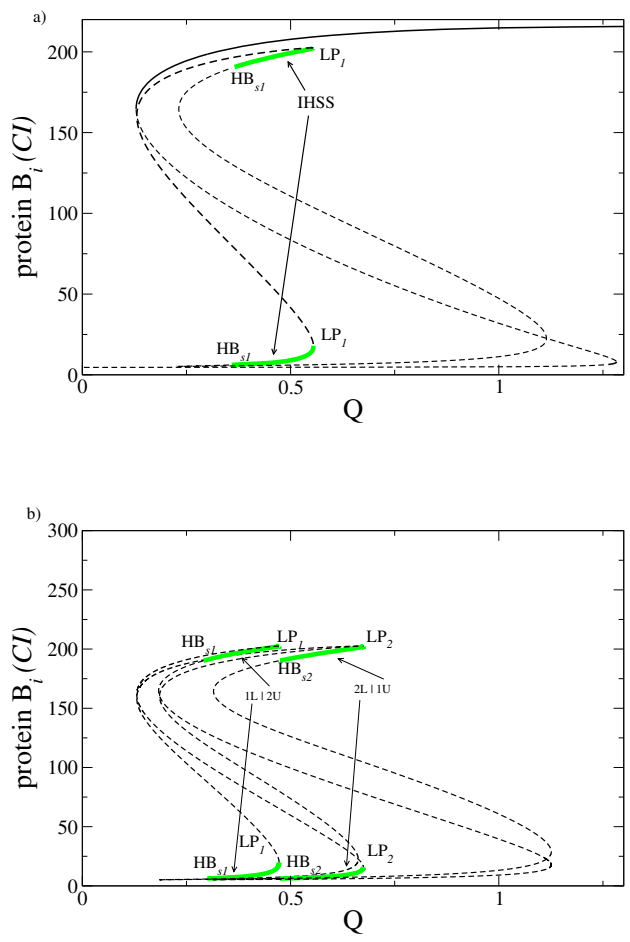

$-\quad-$

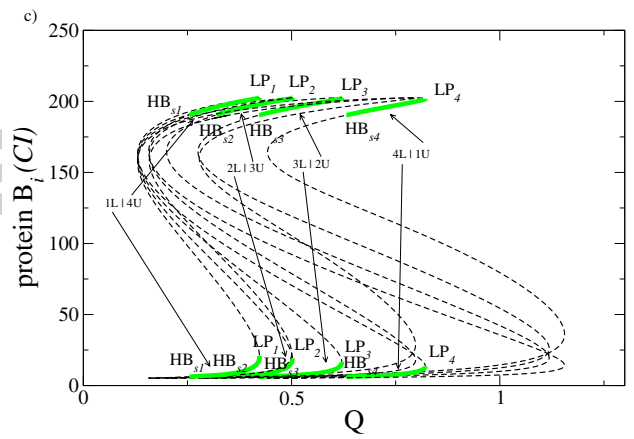

Figure 4: 

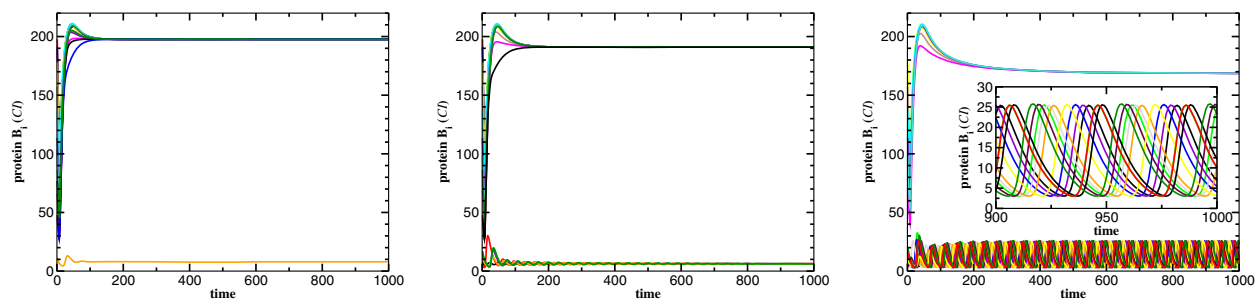

Figure 5: 

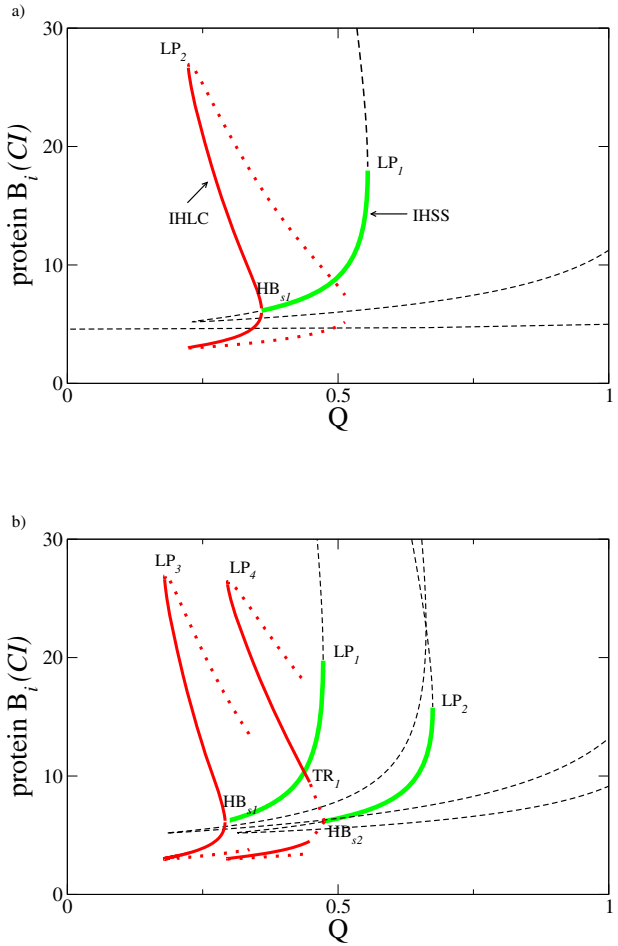

Figure 6: 

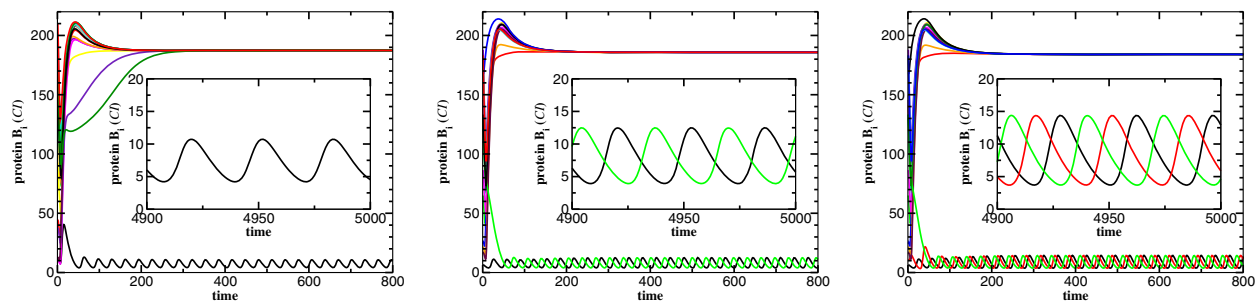

Figure 7: 

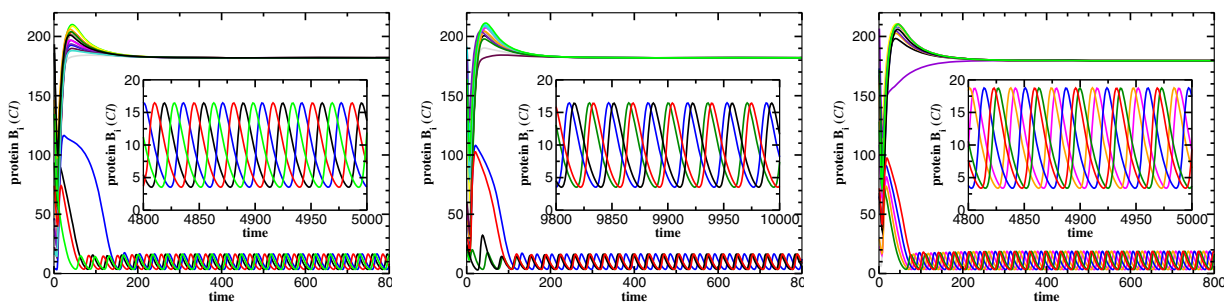

Figure 8: 

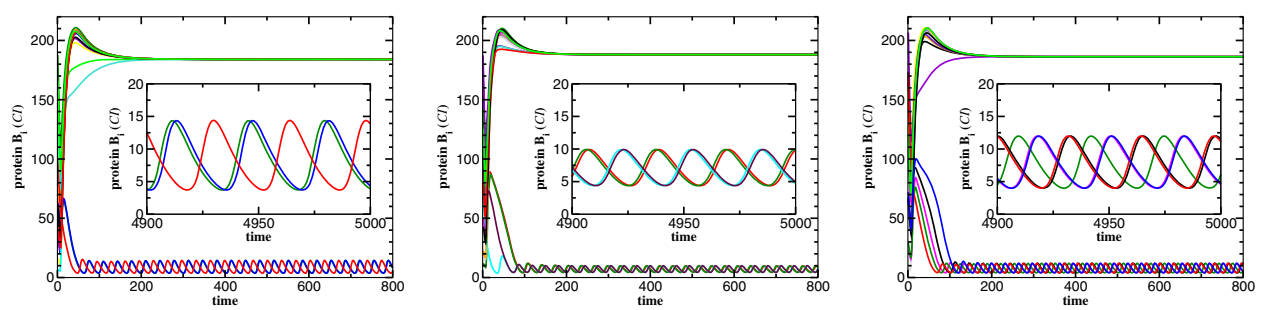

Figure 9: 



Figure 10: 


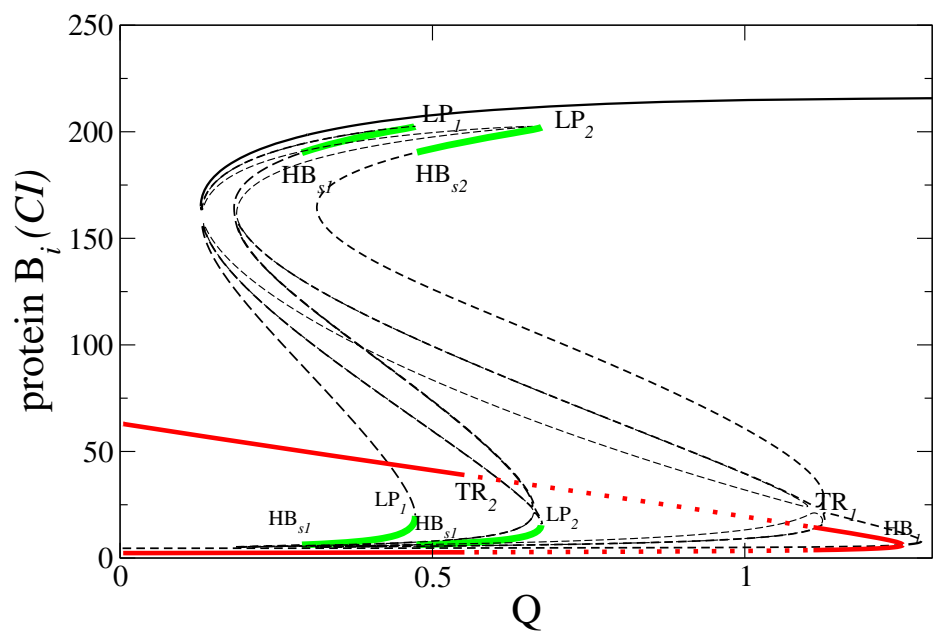

Figure 11: 
a)
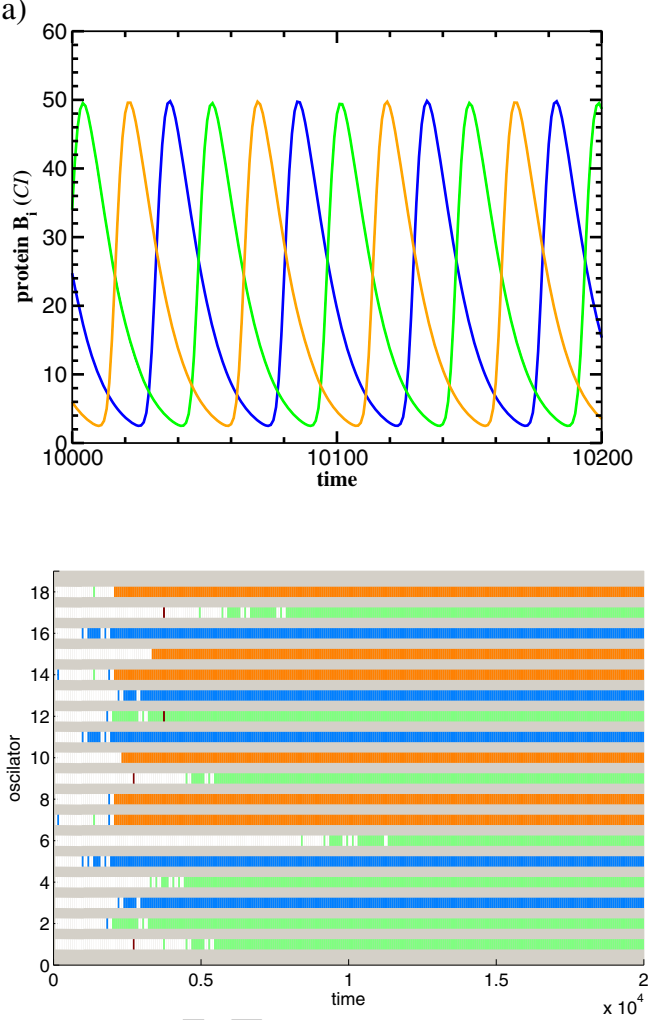

Figure 12: 




Figure 13: 


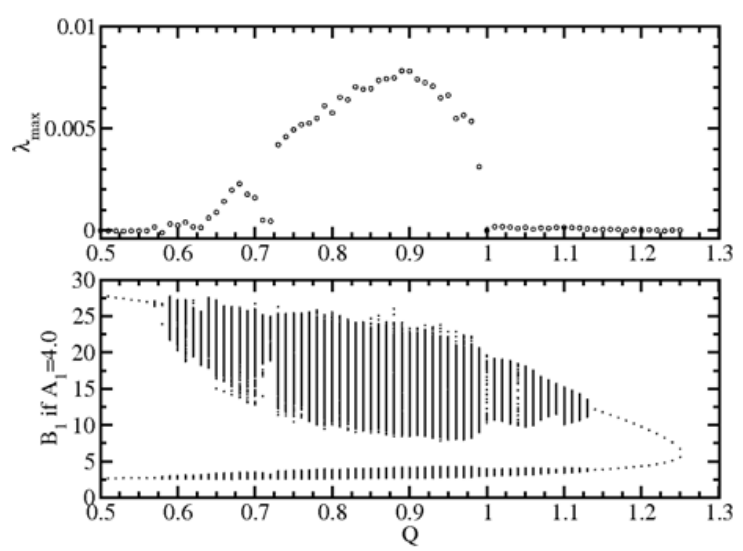

Figure 14: 

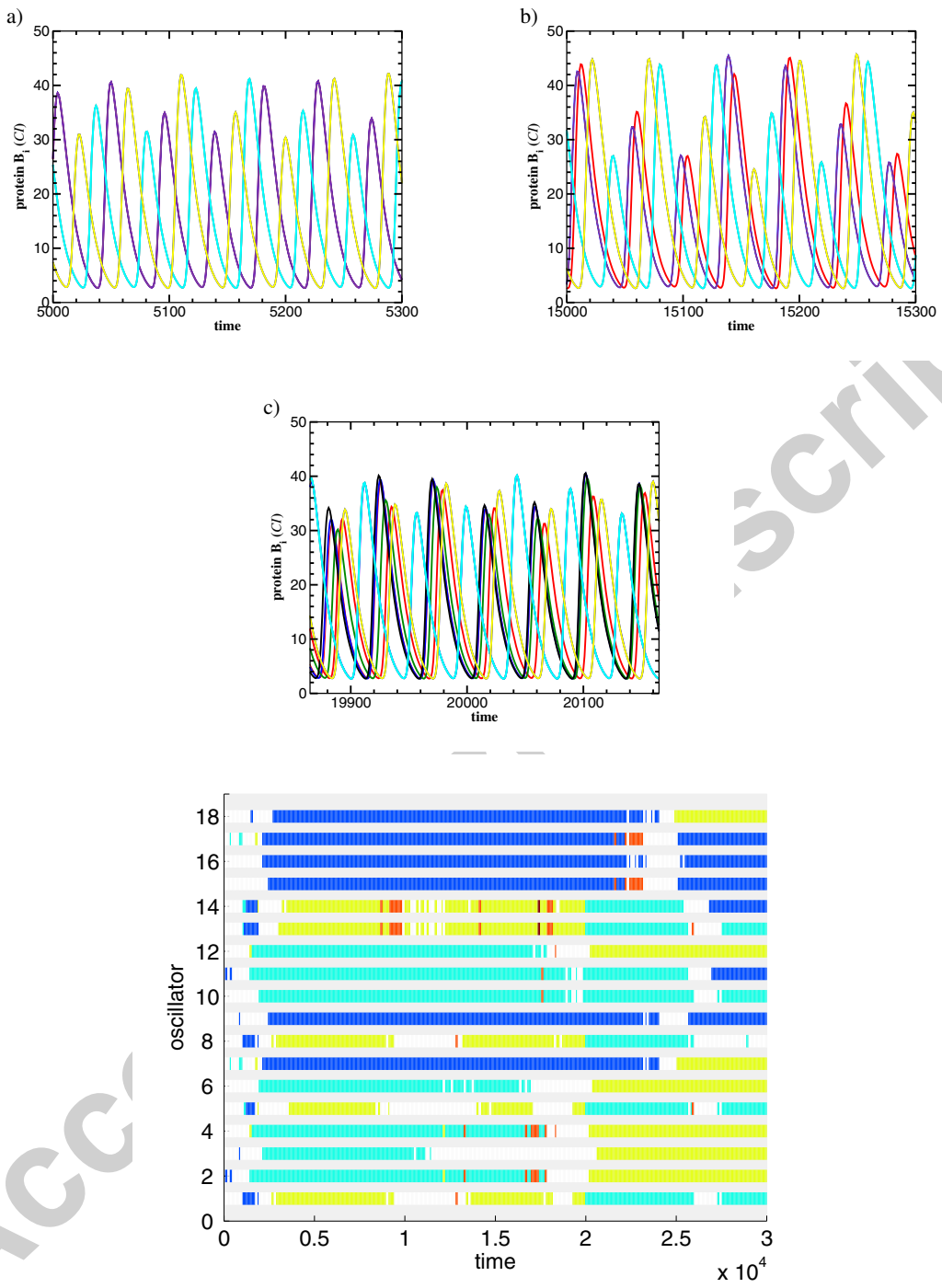

Figure 15: 


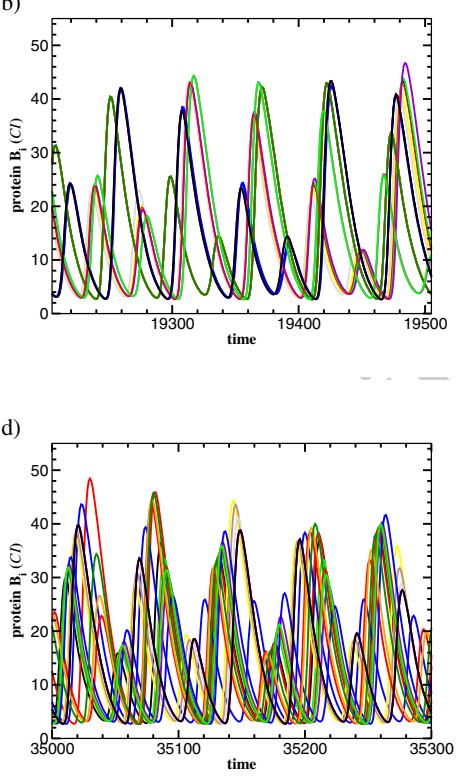

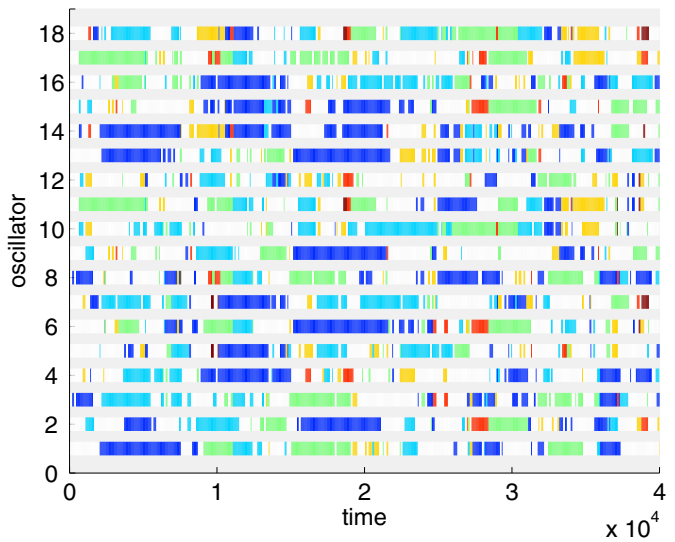

Figure 16: 
747 Figure Legends

748 Figure 1.

749 Scheme of the repressilator with repulsive quorum sensing cell-

750 to-cell communication.

751 Figure 2.

752 Time series for different dynamical regimes for the minimal cou-

753 pled system of $N=2$ : a) $Q=0.4$, homogeneous steady state; b)

$754 Q=0.4$, inhomogeneous steady state; c) $Q=0.1$, full amplitude

755 oscillations and d) $Q=0.3$, inhomogeneous limit cycle. The other

756 parameters are: $n=2.6, \alpha=216, \beta_{a}=0.85, \beta_{b}=0.1, \beta_{c}=0.1, \kappa=25$,

$757 k_{s 0}=1.0, k_{s 1}=0.01, \eta=2.0$.

758 Figure 3.

759 Influence of the system size $(N=2$ in the left plot and $N=18$

760 in the right plot) on the relative regime separation versus coupling

761 strength $Q$. Other parameters as in Fig. 2.

762 Figure 4.

763 Bifurcation chart depicting stable IHSS for: (a) $N=2$; (b) $N=3$

764 and (c) $N=5$ oscillators. Other parameters as in Fig. 2. Here, thick

765 (green) solid lines denote stable IHSS cluster decompositions, and

766 dashed lines denote unstable steady state. Note that the bifurca-

767 tion charts are not complete, depicting only those parts relevant 768 for the current discussion. 
769 Figure 5.

770 Time series of protein $C I\left(B_{i}\right)$ for the IHSS in two different 771 cluster distributions: $1 L \mid U 17$ (left plot) and $6 L \mid 12 U$ (middle 772 plot) and an example of IHLC (right plot) coexisting for the same 773 parameters, with fixed system size $N=18$ and $Q=0.3$. The IHLC 774 example contains 6 oscillators in the upper $C I$ state and 12 in the 775 lower one.

776 Figure 6.

777 Bifurcation structure of the $I H L C$ regime for (a) $N=2$, solid 778 (red) line, and (b) $N=3$ oscillators - the left solid (red) line denotes 779 stable $1 L \mid 2 U$ distribution, whereas the right one denotes stable $2 L \mid$ $7801 U$ distribution. Other parameters as in Fig. 2. Due to the large 781 stiffness of our multidimensional model and the proximity to the 782 bifurcation point, the correct continuation could not be performed 783 with the Xppaut package. Therefore, the bifurcation branches on 784 this figure are not closed.

785 Figure $\%$

786 Examples of different IHLC distributions in an ensemble of $N=$ 78718 cells for the same coupling $Q=0.2$. Every oscillatory sub-cluster 788 consists of only one cell: $1 L \mid 17 U$ (left), $1: 1 L \mid 16 U$ (middle), and $7891: 1: 1 L \mid 15 U$ (right). The insets show a detail of the low-level 790 oscillations after transients. Other parameters as in Fig. 2. 
791

792

793

794

795

796

797

798

799

800

801

802

803

804

805

806

807

808

809

810

811

812

813 Bifurcation diagram of 3 coupled repressilators for increasing

$814 Q$, illustrating the stability of different steady-state and periodic

Figure 8.

I H LC states exhibiting oscillatory low-protein-level sub-regimes with four (left and middle) and five (right) elements in an ensemble of $N=18$ cells. Every sub-cluster in the oscillatory state consists of only one cell. Here $Q=0.2$ and other parameters as in Fig. 2. The left figure depicts the situation with equal phase distance between the oscillating repressilators in the low protein $B$ level, while in the middle and right plots the phase distances are different. The insets belong to the same time series and show a detail of the oscillatory sub-clusters.

\section{Figure 9.}

Examples of IHLC states with complex phase relations between the oscillatory sub-clusters in an ensemble of $N=18$ cells. The insets show in detail the small limit cycle oscillations in the lowerprotein state. Parameters are $Q=0.2$ with $(2: 1) L \mid 15 U$ (left), $Q=0.24$ with $(2: 2) L \mid 14 U$ (middle), $Q=0.24$ with $(2: 2: 1) L \mid 13 U$ (right). Other parameters as in Fig. 2.

Figure 10.

Distribution of dynamical regimes (HSS, IHSS, IHLC) for increasing cell numbers. The coupling strength is fixed to $Q=0.24$ (top) and $Q=0.3$ (bottom). Other parameters as in Fig. 2.

\section{Figure 11.}


815 branches. Due to limitations of the Xppaut package to produce a

816 complete bifurcation diagram of the system, we have used here a

817 small diversity in the $\alpha$ parameter values of different oscillators

818 in the range of $10^{-3}$. This does not qualitatively change the re-

819 sults, but is a sufficient condition to obtain the complete bifur-

820 cation structure of the system. For convenience, we have plotted

821 here only one of the oscillators, although the full analysis has been

822 performed.

823 Figure 12.

824 Top: time series of the protein $B_{i}$ concentration in the regu825 lar oscillating regime, exhibiting three cluster decompositions with $8267: 6: 5$ distribution of cells between them. After a transient, a syn827 chronous behavior inside each cluster emerges and the individual 828 dynamics of the cells inside each cluster are indistinguishable. Bot829 tom: cluster-plot representation of the case above. The parameters 830 are those of Fig. 2, except $Q=0.3$.

831 Figure 13.

832 Cluster plot of $N=11$ non-identical repressilators in the self833 oscillatory regime. A small diversity in parameter $\alpha_{i}$ makes the 834 oscillators non-identical. $\alpha_{i}$ increases from bottom to top. The 835 parameters are: $n=2.6, \alpha_{i}=210,211 \ldots 219,220, \beta_{a}=0.85, \beta_{b}=0.1$, $836 \beta_{c}=0.1, \kappa=25, k_{s 0}=1.0, k_{s 1}=0.01, \eta=2.0$, and $Q=0.5$. 
837 Figure 14.

838 Maximal Lyapunov exponent (top) and a numerical bifurcation 839 plot for the full amplitude oscillations (bottom) for increasing cou-

840 pling $Q$. The simulations have small genetic noise $\sigma_{a}^{2}=10^{-8}$ to avoid

841 tracking unstable orbits. $N=18$, and other parameters are as in

842 Fig. 2.

843 Figure 15.

844 Time series (top panels) and the corresponding cluster plots

845 (bottom panel) in the self-oscillatory regime of $N=18$ oscillators

846 with weak chaotic behavior and long lasting grouping. Parameters

847 are: $n=2.6, \alpha=216, \beta_{a}=0.85, \beta_{b}=0.1, \beta_{c}=0.1, \kappa=25, k_{s 0}=1.0$,

$848 k_{s 1}=0.01, \eta=2.0, \sigma_{a}^{2}=10^{-10}$, and $Q=0.6$.

849 Figure 16.

850 Time series (top panels) and the corresponding cluster plot (bot851 tom panel) in the self-oscillatory regime for $N=18$ oscillators with

852 strong chaotic dynamics and short-lived time grouping, for $N=18$

853 repressilators. The parameters are: $n=2.6, \alpha=216, \beta_{a}=0.85$,

$854 \beta_{b}=0.1, \beta_{c}=0.1, \kappa=25, k_{s 0}=1.0, k_{s 1}=0.01, \eta=2.0, \sigma_{a}^{2}=10^{-10}$, and $855 Q=0.75$. 
Table 1: Examples of the dependence of the IHLC oscillation period on the oscillators distribution between the high- and low-protein levels for $N=18$ and $Q=0.2$.

\begin{tabular}{ccccc}
\hline \hline \# HIGH & \# LOW & PHASE & FIGURE & PERIOD \\
\hline 17 & 1 & - & 7 left & $\approx 31.7$ \\
16 & 2 & equal & 7 middle & $\approx 32.9$ \\
15 & 3 & equal & 7 right & $\approx 34.0$ \\
14 & 4 & equal & 8 left & $\approx 35.3$ \\
14 & 4 & complex & 8 middle & $\approx 35.3$ \\
13 & 5 & complex & 8 right & $\approx 36.5$ \\
\hline \hline
\end{tabular}


Table 2: Examples of the clustering of the full amplitude oscillations.

\begin{tabular}{cccc}
\hline \hline$\underline{Q}$ & $\underline{\text { cluster }}$ & phase & period \\
\hline$N=2$ & & & \\
0.1 & $1: 1$ & equal & 51.3 \\
0.15 & $1: 1$ & equal & 50.0 \\
0.2 & $1: 1$ & equal & 49.4 \\
0.3 & $1: 1$ & equal & 47.7 \\
0.4 & $1: 1$ & equal & 46.0 \\
0.5 & $1: 1$ & equal & 44.5 \\
0.6 & $1: 1$ & complex & many \\
\hline$N=3$ & & & \\
0.1 & $1: 1: 1$ & equal & 51.3 \\
0.15 & $1: 1: 1$ & equal & 50.7 \\
0.2 & $1: 1: 1$ & equal & 50.0 \\
0.3 & $1: 1: 1$ & equal & 48.8 \\
0.4 & $1: 1: 1$ & equal & 47.2 \\
0.5 & $1: 1: 1$ & equal & 46.1 \\
0.6 & $1: 1: 1$ & complex & many \\
\hline & & &
\end{tabular}




\begin{tabular}{cccc}
\hline$N=4$ & & & \\
0.1 & $1: 1: 1: 1$ & equal & 51.3 \\
0.15 & $1: 1: 1: 1$ & equal & 50.7 \\
0.2 & $1: 1: 1: 1$ & equal & 50.1 \\
0.3 & $1: 1: 1: 1$ & equal & 48.8 \\
0.4 & $1: 1: 1: 1$ & equal & 47.6 \\
& $2: 2$ & unstable & - \\
0.5 & $1: 1: 1: 1$ & equal & 46.0 \\
& $2: 1: 1$ & asymmetric & 45.0 \\
0.6 & $2: 2$ & complex & many \\
\hline$N=5$ & & eq & \\
0.0 & - & - & 52.7 \\
0.15 & $2: 2: 1$ & equal & 50.7 \\
0.2 & $2: 2: 1$ & equal & 50.0 \\
0.3 & $2: 2: 1$ & equal & 48.6 \\
0.4 & $2: 2: 1$ & equal & 47.2 \\
0.5 & $2: 2: 1$ & equal & 45.5 \\
& $3: 2$ & unstable & - \\
0.6 & $2: 2: 1$ & equal & 44.1 \\
\hline & & &
\end{tabular}




\begin{tabular}{ccccc}
\hline$N=6$ & & & \\
0.2 & $2: 2: 2$ & equal & 50.1 \\
& $3: 3$ & unstable & - \\
0.3 & $2: 2: 2$ & equal & 48.4 \\
0.4 & $2: 2: 2$ & equal & 47.3 \\
\hline$N=18$ & & & \\
0.2 & $6: 6: 6$ & equal & 50.0 \\
& $7: 6: 5$ & equal & 50.0 \\
& $8: 5: 5$ & equal & 50.0 \\
& $7: 7: 4$ & unstable & - \\
& $8: 6: 4$ & unstable & - \\
& $9: 9$ & unstable & - \\
\hline$N=100$ & & & \\
0.4 & $34: 34: 32$ & equal & 47.2 \\
& $35: 33: 32$ & equal & 47.2 \\
& $35: 34: 31$ & equal & 47.2 \\
& $36: 33: 31$ & equal & 47.1 \\
\hline \hline
\end{tabular}

